\title{
3. Forschungsstand
}

Der Forschungsstand zur politischen Steuerung von barrierefreiem bzw. -reduziertem Wohnraum ist äußerst überschaubar. Am nächsten kommt meinem Forschungsvorhaben die Dissertation von Joo (2018), welche sich mit Wohnberatungen zur altersgerechten Wohnungsanpassung beschäftigt und hier auch konkret politische Aktivitäten untersucht. Zudem bietet der Wohnatlas des Kuratoriums Deutsche Altershilfe in Kooperation mit der Wüstenrot Stiftung einen wertvollen Startpunkt, arbeitet er doch verschiedene politische Maßnahmen zum »Wohnen im Alter« strukturiert auf (KDA/Wüstenrot Stiftung 2014a; KDA/Wüstenrot Stiftung 2014b). Ähnliches gilt für eine gleichnamige Studie des Bundesbauministeriums (BMVBS 2011). Die politischen Prozesse, die zu diesen Maßnahmen geführt haben, wurden jedoch bislang nicht wissenschaftlich untersucht.

Die vorliegende Forschungsarbeit verortet sich in der Politikfeldanalyse und den einschlägigen politikwissenschaftlichen Arbeiten zur Wohnungspolitik. Dafür ebnet Kapitel 3.1 zunächst den Weg in die politikfeldanalytische Auseinandersetzung mit der Untersuchungsebene - den Bundesländern. Aus dieser Literatur können nicht nur Erkenntnisse zu bewährten Methoden und Forschungsdesigns, sondern auch grundlegende Strukturen und Handlungslogiken auf Länderebene abgeleitet werden. Kapitel 3.2 arbeitet daraufhin die politikwissenschaftliche Literatur zur Wohnungspolitik auf, um das Forschungsvorhaben inhaltlich zu verankern. Zuletzt erfolgt mit Kapitel 3.3 eine Zusammenfassung und Reflexion des aufgearbeiteten Forschungsstandes.

\subsection{Bundesländer in der Politikfeldanalyse}

Um die Bedeutung der Bundesländer als Untersuchungsgegenstand in der Politikfeldanalyse zu verdeutlichen, interessiert zunächst, welche landespolitischen Politikfelder hier von anderen Policy-Forscher*innen beforscht werden und wo es Forschungslücken gibt. Wird eher vergleichend oder über Einzelfallstudien vorgegangen? Die aufgearbeitete Studienlage basiert zu einem großen Teil auf Sammelbänden zur Politik der Bundesländer (Hildebrandt/Wolf 2016; Freitag/Vatter 2010; Scheller/Schmid 2008; Schnei- 
der/Wehling 2006) sowie auf einem Schwerpunktheft der Zeitschrift für Vergleichende Politikwissenschaft (Sack/Töller 2018). Der Blick in diese Literatur offenbart eine Schwerpunktsetzung auf vergleichend angelegte Studien in einzelnen Politikfeldern.

In den klassisch unter die legislative Zuständigkeit der Länder fallenden Politikfeldern ist die Literaturbasis naturgemäß besonders breit. Dies gilt für die Bildungspolitik, wozu sowohl Schulpolitik (Ulbricht 2014; Hepp 2006; Wolf/Heinz 2016) als auch Hochschulpolitik zählt (Lanzendorf/Pasternack 2016). Ferner stellt Münch (2008) die Frage nach einer »bundesdeutschen Bildungspolitik« trotz föderaler Landeszuständigkeiten und Schlicht (2010) untersucht die Wechselwirkungen zwischen Bildungspolitik und Bildungsungleichheit. Ebenfalls intensiv beforscht ist die Arbeitsmarktpolitik der Länder (grundlegend Blancke/Schmid 1998). Während Blancke/Schmid (2006) und Stoy (2016b) sich in ihren Analysen für eine Verbindung von Arbeits- und Sozialpolitik entscheiden, untersucht Blancke (2004) die Diffusion unterschiedlicher Arbeitsmarkt-Policies und Malik (2008) fragt nach den Auswirkungen der Hartz IV-Reformen auf die Arbeitsmarktpolitik der Länder. An die Arbeits- und Sozialpolitik angrenzend sind verschiedene Studien im weiteren Sinne der Sozial- und Gesellschaftspolitik zuzuordnen, wie etwa Jeffery et al. (2014) zur Diversität von Lebensbedingungen oder in der Gesundheitspolitik sowohl Böhm et al. (2018) zur Umsetzung des Präventionsgesetzes als auch Gerlinger (2008) zu den Bund-Länder-Beziehungen im entsprechenden Politikfeld. Stoy (2016a) und Schieren (2008) interessieren sich indessen stärker für einen breiteren Ansatz der föderalen Sozialpolitik - je unter dem Leitsatz des unitarischen Bundesstaats. Und während sich Beinborn et al. (2018) mit der Ländervarianz in den Jugendpolitiken ein eher exotisches Politikfeld vornehmen, sind verschiedene Arbeiten der Integrationsund Kulturpolitik zuzuordnen (von Blumenthal 2010; Stocker 2010; Münch 2016; Günther et al. 2019).

Die Umwelt-, Klima- und Energiepolitik bildet einen weiteren Schwerpunkt in der Politikfeldanalyse auf Ebene der Bundesländer. Der Landesumweltpolitik wird sich genähert entlang von Fragestellungen zur Umsetzung europäischer Vorgaben (Eppler 2008), zu Wahlprogrammen (Seeger 2003), in Verbindung zur Naturschutzpolitik (Böcher/Töller 2016) oder über vergleichende Umweltindikatoren (Berker et al. 2019). Die Energiepolitik erarbeiten Wurster/Köhler (2016) in ihrer allgemeinen Struktur, wohingegen Monstadt/Scheiner (2016) stärker die legislative Aufgabenverteilung interessiert. Kerkow (2016) schließlich untersucht die Umsetzung der Agenda 2030 der Vereinten Nationen in den einzelnen Bundesländern. Ferner findet auch der landespolitische Komplex Sicherheitspolitik, Polizei und Justiz regen Anklang in der Policy-Forschung (Frevel/Groß 2016; Rowe/Turner 2016; Prätorius 2006; Bandau/Bothner 2020). Hier erklären Stoiber/Töller (2016) die Privatisierung vom Maßregelvollzug und Schniewind et al. (2010) vergleichen die Landesausgaben der Sicherheitspolitik mit der Bildungspolitik. Wie Polizei und Justiz auch ist die Verwaltungspolitik eine typische Landeskompetenz. Insofern erscheint es nur folgerichtig, dass sich zahlreiche Arbeiten mit diesem Politikfeld und insbesondere mit den darin stattfindenden Verwaltungsreformen beschäftigen (Bogumil 2007; Haus 2006; Ebinger/Bogumil 2016; Grotz et al. 2017).

Die landesspezifischen Strukturen der Wirtschafts- und Finanzpolitik (Hildebrandt 2016; Reus 2016) interessiert die Policy-Community ebenso wie die in diesem Politikfeld bestehende Verflechtung zwischen Bund und Ländern (Scheller 2008; Woisin 2008). 
Das Stichwort der Verflechtung ist naturgemäß ebenfalls zentral bei der Europa- und Bundespolitik der Länder: Während Grasl (2016) hierfür als breiterer Überblick zu empfehlen ist, konzentriert sich Hüttmann (2008) auf die Beziehung zur Bundesebene. Andere Autor*innen stellen wiederum die Europäisierung in den Mittelpunkt ihres Interesses (Hüttmann/Siegl 2008; Derpa 2008; Sack/Sarter 2018) oder fragen nach der Umsetzung europäischer Vorgaben durch die Bundesländer (etwa Ulbricht/Römer 2019 zur Datenschutzaufsicht).

Schließlich sei auf einige Arbeiten verwiesen, welche entweder keinem klassischen landespolitischen Kompetenzbereich zugeschrieben werden können, sich erst in den letzten Jahren entwickelt haben oder aus anderen Gründen bislang keine sonderliche Aufmerksamkeit in der Policy-Literatur erfahren. Für das vorliegende Erkenntnisinteresse besonders anregend ist die Arbeit von Holtmann (2005) zur dezentralen Aufgabenverteilung in der Bau- und Wohnungspolitik - allerdings noch vor der Föderalismusreform 2006. Weiterhin untersucht Lange (2006) die Medienpolitik der Länder und Schwedes/Ruhrort (2016) interessiert die Verkehrspolitik - ein angesichts der empirischen Relevanz ebenfalls erstaunlich dünn besetztes Politikfeld in der einschlägigen Literatur. Die Regionalpolitik, insbesondere zur regionalen Entwicklung von ländlichen Räumen, betrachtet Ewert (2016) in Verbindung mit der Landwirtschaftspolitik, wohingegen Grajewski/Mehl (2008) sich für darin ausbildende Netzwerkstrukturen interessieren und Kilper (2008) nach Anpassungsleistungen in der Regionalpolitik fragt. Ebenfalls die Regionalpolitik, dabei in Verbindung zur Stadtentwicklung und Städtebauförderung, untersucht Zimmermann (2016). Aufgrund der Nähe der Wohnungspolitik zur Städtebauförderung erscheint auch hier ein tieferer Blick lohnend.

Die bislang dargestellten Studien sind allesamt vergleichend angelegt, was bei der Ausgangslage von 16 Bundesländern mit ähnlichen institutionellen Rahmenbedingungen nicht verwundert. Nichtsdestotrotz finden sich auch Fallstudien zu einzelnen Politikfeldern bzw. Policies in einzelnen Bundesländern (etwa: Hörisch/Wurster 2017 für Baden-Württemberg; Reutter 2013 sowie Wiegand 2015 für Nordrhein-Westfalen). Diese inhaltlich breite Literatur kann hier nicht umfassend aufgearbeitet werden, wird aber im weiteren Verlauf insbesondere bzgl. der Fallstudienländer Hessen und SachsenAnhalt von Interesse sein (vgl. Stoiber et al. 2007; Hornig 2016). In diesem Kontext sei auch auf die Sammelbände zur politischen Landeskunde in einzelnen Bundesländern verwiesen. Mit je eigenen Kapiteln zu den landesspezifischen Institutionen, Akteuren und politischen Kulturen - aber teils auch bereits mit Blick in einzelne Politikfelder bieten sie eine wertvolle Literaturbasis zur Annäherung an landespolitische Fragestellungen. Insbesondere wichtig sind für die Fallstudien dabei »Politik und Regieren in Sachsen-Anhalt« (Träger/Priebus 2017) sowie »Politik und Regieren in Hessen« (Schroeder/Neumann 2016a).

Abschließend bleibt zur einschlägigen Studienlage festzuhalten: Policy-Forscher*innen untersuchen die Ebene der Bundesländer durchaus und das nicht erst seit gestern. Dass dies auch seine empirische Berechtigung hat, stellt Gebauer (2010: 245) fest:

»Entgegen einer weit verbreiteten [...] Auffassung verfügen die Länder nämlich durchaus über zentrale, relevante und zukunftsträchtige Gesetzgebungskompetenzen. [...] 
Jedenfalls kann heute noch weniger als vor einigen Jahren die Rede davon sein, dass den Ländern keine substanzielle Gesetzgebungsmacht verblieben wäre«.

Die Wohnungspolitik ist indessen in der Policy-Forschung der Bundesländer bislang, wenn überhaupt, nur marginal vertreten. Dies überrascht angesichts der legislativen Zuständigkeiten doch sehr (vgl. Kapitel 2.1). Eine Erklärung mag sein, dass die Wohnungspolitik zwischenzeitlich nicht mehr als eigenständiges Politikfeld, sondern als Bestandteil der Stadtentwicklungspolitik verstanden wird bzw. wurde. Dabei ist auch dieser Bereich - mit wenigen Ausnahmen wie Zimmermann (2016) - politikwissenschaftlich nur wenig erforscht. Eine andere mutmaßliche Erklärung liegt in der langjährigen politischen und gesellschaftlichen Geringschätzung dieses Politikfeldes (vgl. Kapitel 1): Folglich war auch die wissenschaftliche Aufmerksamkeit eine randständige. Sollte diese Vermutung zutreffen, so ist ab Mitte der 2o10er Jahre - dabei je die zeitlichen Verzögerungen vom Forschungsinteresse bis hin zur Publikation berücksichtigend - eine verstärkte Publikationsaktivität zu erwarten.

Die Erkenntnisse aus anderen Politikfeldern bieten aber dennoch eine wertvolle Ausgangsbasis. Erstens verhelfen sie zu einem tiefergehenden Verständnis landespolitischer Prozesse und zweitens sind insbesondere aus der Sozial- und Arbeitsmarktpolitik, aber auch aus der Wirtschafts- und Finanzpolitik, Übertragungen zum verwandten Bereich der Wohnungspolitik denkbar.

\subsection{Wohnungspolitik in der Politikwissenschaft}

Nachdem die politikfeldanalytische Literatur zu den Bundesländern als Untersuchungsebene dargestellt wurde, verengt sich das folgende UnterKapitel inhaltlich auf die Wohnungspolitik. Diesem Politikfeld ist der Bereich des barrierefreien bzw. -reduzierten Wohnraums zuzuordnen. Dabei scheint die politikwissenschaftliche Beschäftigung mit der Wohnungspolitik dem Takt der politischen Aufmerksamkeit nahtlos zu folgen: Während die 200oer und frühen 2010er Jahre eine weitgehend publizistische Abstinenz kennzeichnete, verstärkt sich in den letzten Jahren die Forschungsaktivität. ${ }^{1}$

Wie lässt sich der Forschungsstand zu »der Wohnungspolitik« kategorisieren? Denkbar wäre eine Strukturierung entlang der föderalen Untersuchungsebenen, allerdings widerspricht dieses Vorgehen der Publikations- und Denklogik vieler Autor*innen. Diese sind zumeist an einzelnen Problemen oder Policies interessiert unabhängig davon, welche politische Ebene je über Handlungskompetenzen verfügt. Vielfach lassen sich wohnungspolitische Herausforderungen ohnehin nur im Mehrebenensystem bearbeiten. Vor diesem Hintergrund wird zunächst der bestehende politikwissenschaftliche Forschungsstand zur allgemeinen Wohnungspolitik aufgezeigt. Daraufhin sind diejenigen wohnungspolitischen Debatten zu erörtern, denen die Politikwissenschaft aktuell ihre Aufmerksamkeit widmet.

1 Aufgrund der nationalstaatlich stark variierenden Wohnungsmärkte und Wohnungspolitiken beschränkt sich dieses Kapitel auf die deutsche Wohnungspolitikforschung. 